\title{
Influence of Rayleigh-Bénard convection on electrokinetic instability in overlimiting current conditions
}

\author{
Joeri C. de Valença, ${ }^{1,2, *}$ Aziz Kurniawan, ${ }^{2}$ R. Martijn Wagterveld, ${ }^{2}$ Jeffery A. Wood, ${ }^{1}$ \\ and Rob G. H. Lammertink ${ }^{1, \dagger}$ \\ ${ }^{1}$ Soft Matter, Fluidics and Interfaces Group, MESA ${ }^{+}$Institute of Nanotechnology, University of Twente, \\ 7500AE Enschede, The Netherlands \\ ${ }^{2}$ Wetsus, European Centre of Excellence for Sustainable Water Technology, Oostergoweg 9 , \\ 8911MA Leeuwarden, The Netherlands \\ (Received 8 August 2016; published 31 March 2017)
}

\begin{abstract}
We investigate the influence of buoyancy on electroconvection at an ion-exchange membrane in an aqueous electrolyte solution. Electrokinetic instabilities (EKIs) and Rayleigh-Bénard (RB) convection are both known to mix the appearing concentration gradient layer and overcome the limiting current arising from diffusional limitations. The different physics, as well as the interplay between them, are investigated by electrical, flow, and concentration characterization. In the buoyancy stable orientation, an EKI mixing layer, having a low concentration, grows till saturated size. In the buoyancy unstable orientation, $\mathrm{RB}$ occurs and dominates the advective transport due to the large system size. When current density $i<5 i_{\lim }$, RB mixes the system and EKI does not arise. If $i>5 i_{\lim }$ EKI starts before $\mathrm{RB}$ and hastens the onset of RB. Upon onset of RB, EKI is suppressed while the overall resistance is still decreased. The onset times of EKI and RB could be predicted using a simple diffusion-migration model based on Fick's second law.
\end{abstract}

DOI: 10.1103/PhysRevFluids.2.033701

\section{INTRODUCTION}

For charge-selective interfaces such as ion-selective membranes or micro-nanochannel systems with Debye layer overlap, the existence of the so-called "limiting current," $i_{\text {lim }}$, is a well-known theoretical and experimentally established phenomena (see [1,2], for example). Currents above $i_{\text {lim }}$, called overlimiting current (OLC), have been observed experimentally and are related to additional transport mechanisms or water dissociation [1,3-5]. Electrically induced advection through electroosmotic forces near the membrane interface in an extended space charge layer is a known mechanism behind OLC. The scientific path of theoretical prediction [6,7] to experimental visualization [8-10] and more recent direct numerical simulations [11-13] has brought an understanding to the set of criteria that is needed to start this electrokinetic instability (EKI). Further research has been done on the three-dimensional (3D) nature of EKI [14,15], as well as the coupling with pressure-driven flow $[15,16]$ and the modification of the membrane surface $[17,18]$. Previously, we have investigated the coupling of electrokinetic instabilities to observed electrical signals, characterizing and quantifying the transient dynamics of fluid vortices that arise [3].

Advective mixing can also be driven by buoyancy. When a less dense (ion-depleted) fluid lies below a denser (ion-enriched) fluid, gravitational forces can start mixing within the fluid. If the density gradient is parallel to the gravitational field, then mixing will occur above a critical concentration (or density) gradient, identified with the Rayleigh number, called Rayleigh-Bénard (RB) convection [19-21]. At electrodes during electrodeposition, the morphology of the metal deposit is known to change due to RB convection [22-24]. Buoyancy mixing of the boundary layer near a membrane is known to decrease the mass transfer resistance [25-27]. Recently, direct numerical simulations

*joeri@valenca.nl

†r.g.h.lammertink@utwente.nl 


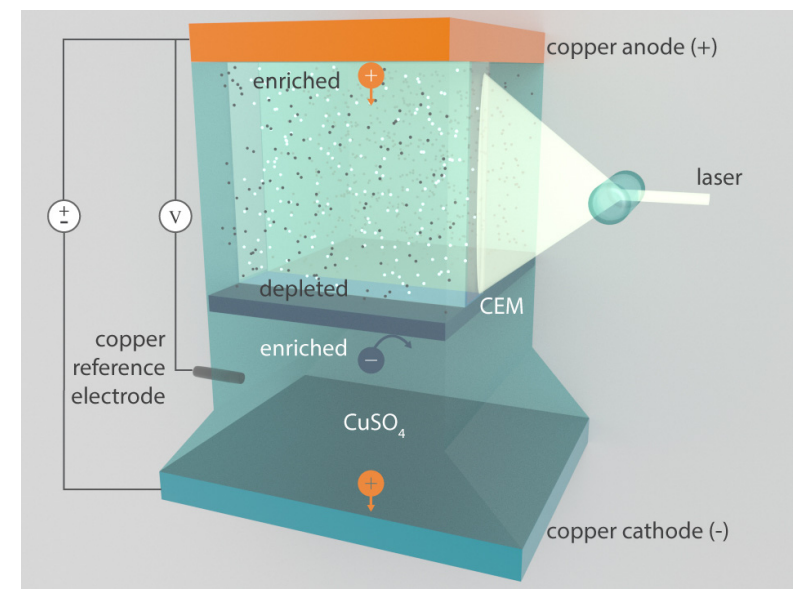

FIG. 1. Experimental setup in co-orientation, the buoyancy unstable situation, where the electric and gravitational field point downwards, noted as $\mathrm{g} \downarrow \mathrm{E} \downarrow$. The voltage drop, $\Delta V$, under a constant applied current, $I$, is measured simultaneously with the local hydrodynamics of the ionic solution inside the anode compartment.

to study the coupling of RB and EKI near a membranes have been performed [28]. The estimated currents were greatly influenced by the appearance of RB convection. No universal relation could be obtained for the current, as the RB and EKI were predicted to be coupled in highly nonlinear fashion.

In this paper, we report our experimental investigations on the influence of Rayleigh-Bénard convection, the electrokinetic instability, and coupling between these phenomena in a cationexchange membrane system. We experimentally quantified the changing concentration profile, flow, and electrical response of the setup for different orientations of electric field with respect to the gravitational field. This is accomplished using fluorescence lifetime imaging microscopy (FLIM), particle image velocimetry (PIV), and potentiostatic measurements. From these dynamic measurements, we also compare convection onset times to a simple transient model based on Fick's law of diffusion.

\section{MATERIAL AND METHODS}

\section{A. Electrochemical measurements (chronopotentiometry)}

Figure 1 shows the experimental setup, composed of a cation-exchange membrane, (Neosepta CMX), with surface area $A_{\text {mem }}=3 \times 4.5 \mathrm{~mm}^{2}$, and thickness of $170 \mu \mathrm{m}$, placed between two reservoirs filled with a $10 \mathrm{mM} \mathrm{CuSO}$ electrolyte solution, both closed with copper electrodes. The distance between membrane and anode is $2 \mathrm{~mm}$ and between membrane and cathode $20 \mathrm{~mm}$. Chronopotentiometric measurements are performed by forcing a constant dc electric current, $I$, through the setup (Autolab PGSTAT 30). The anode, where copper oxidizes, serves as a $\mathrm{Cu}^{2+}$ cation source, and the cathode, where copper reduces, acts as a $\mathrm{Cu}^{2+}$ cation sink. The time-dependent voltage difference, $\Delta V(t)$, was measured between the anode and a copper wire placed on the other side of the membrane $(7.3 \mathrm{~mm}$ from membrane). The current density was set between $i=4.4-44.4 \mathrm{~A} / \mathrm{m}^{2}$.

The ion-transport process is investigated for two different orientations. (1) The membrane is placed horizontally with the anode compartment above it (Fig. 1). This is called co-orientation, since the electric field direction (anode to cathode) is aligned with the gravitational field (buoyancy unstable). (2) The setup is rotated $180^{\circ}$, which we call counterorientation (buoyancy stable). 


\section{B. Flow measurements (PIV)}

Flow dynamics in the anode compartment are measured by seeding the solution with $0.1 \mathrm{wt} \% 2-\mu \mathrm{m}$ red polystyrene tracer particles (Microparticles $\mathrm{GmbH}$, with a density of $1.05 \mathrm{~g} / \mathrm{cm}^{3}$ ). The particles are illuminated with a thin laser sheet (Firefly, Oxford lasers) (see Fig. 1), and the reflected light is captured through a long-distance magnifying lens (Navitar) on a camera at 10 or 32 frames per second. From the recorded particle displacement, we determine the vector field using particle image velocimetry (PIV) analysis (DaVis) [Figs. 3(b) and 3(e)].

Image recording. Depending on the particle velocity and magnification, an appropriate recording frequency is chosen. The movie and PIV analysis in of the mixing layer in the counterorientation at $29.6 \mathrm{~A} / \mathrm{m}^{2}$ was done using $\Delta t=0.03 \mathrm{~s}$. All other movies and PIV analysis are done using $\Delta t=0.1 \mathrm{~s}$. The typical magnification of the magnifying lenses attached to the camera is $M=2.5$ (with the resolution of 1 pixel $=2 \mu \mathrm{m}$ ). The entire compartment, $2 \mathrm{~mm}$ in height and $3 \mathrm{~mm}$ in width, is in view.

Multigrid vector calculation. To calculate the flow field, a multigrid cross-correlation method with decreasing window size is used. First, a $98 \times 98$-pixel interrogation window is used to determine a reference vector field. This field is then used to calculate a window shift of the next correlation. This shift ensures the same particles are correlated with each other. To obtain a higher resolution, the second calculation is done with windows of $48 \times 48$ pixel. A geometrical mask covers the membrane, anode, and side wall so no vector field is calculated in that region.

Vector postprocessing. To eliminate erroneous vector, a vector postprocessing algorithm is used. Outlier detection is employed based on the median value of the nearest neighbors. This value is less sensitive to neighboring outliers with large values compared to a normal average. If appropriate, a new vector is calculated and groups of erroneous vectors can also be excluded. This scheme consists of the four following steps. First, vectors outside the allowed deviation from the neighbors are eliminated using the criteria

$$
\begin{gathered}
U_{\text {median }}-a U_{\mathrm{rms}} \leqslant U \leqslant U_{\text {median }}+a U_{\mathrm{rms}}, \\
U_{\mathrm{rms}}=\sqrt{\frac{1}{n} \sum_{i=1}^{n}\left(U_{i}-U_{\text {median }}\right)^{2}}
\end{gathered}
$$

where $a=2$. This criterion is used for both $x$ and $y$ components of a planar velocity vector $(u, v)$ to check the validity of the velocity vector. In the second step, all vectors with less than three neighbor vectors are also removed. In the third step, new vectors for the empty vector positions are found using an iterative process. For vectors with three or more neighbors, the median criterion is checked with $a=3$. If the vector is identified as false, a lower correlation peak is checked for fitting up to the fourth-lowest peak. Once no more vectors can be added, the final step effectively detects and subsequently removes small groups of spurious vectors (with less than four vectors) which have not been detected in the steps 1 and 2. If no vectors meet these criteria in the integration windows, the vectors in the empty spaces are interpolated or extrapolated. The average values of $u$ and $v$ of the nonzero nearest neighbors are determined and subsequently used. This procedure is done iteratively to fill up the whole grid. The dynamics are relatively slow, so for five consecutive vector fields the orthogonal components are averaged separately (sliding average) to reduce the influence of erroneous vectors further. The final vector fields represent the average motion during $\Delta t=0.5 \mathrm{~s}$, or $\Delta t=0.16 \mathrm{~s}$ in case of $29.6 \mathrm{~A} / \mathrm{m}^{2}$ in the counterorientation.

Determining vortex size and speed. In co-orientation, motion occurs in the full cell and the root mean square of all vectors of the final vector field is taken as the mean velocity of the mixing layer. In counterorientation, first the mixing layer thickness $\left(L_{\text {mix }}\right.$ or vortex size) needs to be determined from the final vector field before the vortex speed (root mean square velocity in the vortex area) can be calculated. The vortex boundary is characterized by the sharp decay of the vertical velocity 

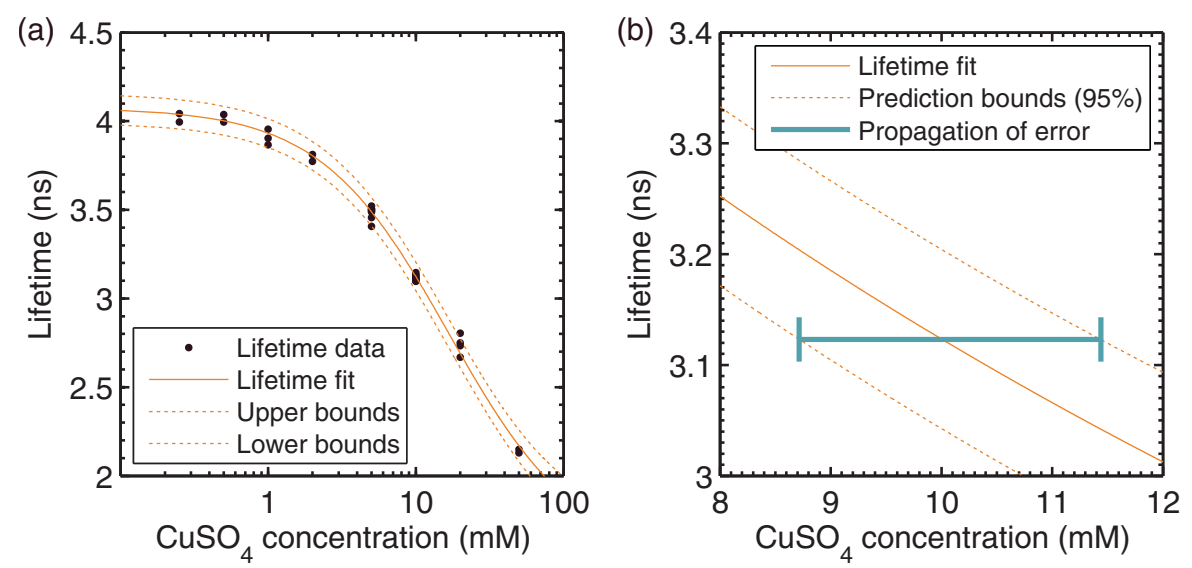

FIG. 2. (a) FLIM calibration curve on semilog scale. (b) From the prediction bounds, an estimate on the inverse error of determining concentration from lifetime can be determined.

component, $v$, away from the membrane. From a horizontal row (40 vectors) the average value is taken. These average values are interpolated with a cubic spline to get a smooth function representing the change in vertical velocity. The vortex region is quantified by the distance between the membrane position (determined with IMAGEJ) and the position where the vertical velocity drops below a fixed threshold value. The threshold value is taken as $10 \%$ of the maximum value inside the vortex region. The absolute value of the vectors in this vortex region is averaged to obtain the vortex speed. A similar technique was used in our previous paper [3].

\section{Concentration measurements (FLIM)}

The concentration profile in the system is determined using FLIM(LaFLIM, Lambert Instruments, Groningen, The Netherlands). The fluorescence lifetime of Alexa Fluor 488 dye depends on the $\mathrm{CuSO}_{4}$ concentration in the range between 1 and $100 \mathrm{mM}$, as seen in the calibration curve and error estimation (see Fig. 2). A modulated blue light-emitting diode light is sent through a $5 \times$ magnifying objective (Zeiss) and the fluorescence is captured on a $696 \times 520$-pixel CCD camera. Each pixel represents an area of $4.17 \times 4.17 \mu \mathrm{m}^{2}$. To have sufficient light intensity, each image was made with a shutter time of $\Delta t=300 \mathrm{~ms}$. From 12 phase images the shift of fluorescence lifetime of each pixel is determined compared to a reference images. The reference is demineralized water (Milli-Q) with $2.5 \mu \mathrm{M}$ Alexa Fluor 488 dye. The lifetime of that sample is set to $4.1 \mathrm{~ns}$, according to literature $[29,30]$ and confirmed by a fluorescein reference lifetime standard.

The fluorescence decay of Alexa Fluor 488 dye is concentration dependent in the range between 1 and $100 \mathrm{mM}$ [see Fig. 2(a)]. A modified Stern-Volmer equation was used to fit the fluorescence lifetime $\tau$ to the $\mathrm{CuSO}_{4}$ concentration $c$ [31]:

$$
\tau=A\left(1-\frac{1}{\frac{1}{f K c}+\frac{1}{f}}\right) .
$$

The least-squares parameter estimates of this function were $A=4.0761 \pm 0.0263 \mathrm{~ns}, K=0.0598 \pm$ $0.0058 \mathrm{mM}^{-1}$, and $f=0.6243 \pm 0.0250$. $A$ corresponds (within error) to the expected lifetime of Alexa dye in water $(\sim 4.1 \mathrm{~ns})$ [30]. From the confidence intervals the prediction bound of $95 \%$ is determined. Once the lifetime is measured, the error bar in concentration is derived via this prediction bound [see Fig. 2(b)]. Normality of residuals was assessed using visual inspection of a normal probability plot. 


\section{Numerical modeling of concentration profile}

The degree of concentration polarization in the anode compartment is predicted using a transient 1D formulation of Fick's second law. This follows from combining the Nernst-Planck equation with the continuity relation and electroneutrality assumption Ref. [32]. The membrane and anode are assumed to be ideally cation selective. The initial condition is a homogeneous concentration and the boundary conditions are set by the applied constant current. At the anode there is a constant inflow of cations and at the membrane a constant outflow. The development of the concentration gradients are solved via a partial differential equation (PDE) solver (MATLAB); an analytical solution can also be found in [32]. This allows for prediction of concentration gradient and onset times of EKI and $\mathrm{RB}$ in the cell. The equations read

$$
\frac{\partial c(x, t)}{\partial t}=D \frac{\partial^{2} c(x, t)}{\partial x^{2}}
$$

with the initial and boundary conditions

$$
\begin{gathered}
c(x, 0)=c_{0}, \\
\frac{\partial c(0, t)}{\partial x}=\frac{i}{z F D}\left(\overline{t_{+}}-t_{+}\right), \\
\frac{\partial c(L, t)}{\partial x}=\frac{i}{z F D}\left(\hat{t_{+}}-t_{+}\right),
\end{gathered}
$$

where $c(x, t)$ is the concentration, $z$ is the valency of the (symmetric) electrolyte $(z=2), F$ is Faraday constant $\left(F=9.65 \times 10^{4} \mathrm{C} / \mathrm{mol}\right)$, and $D$ is the diffusion coefficient $\left(D_{\mathrm{CuSO}_{4}}=0.855 \times 10^{9} \mathrm{~m}^{2} / \mathrm{s}\right.$ [33]). We assume an ideal membrane and electrode that only allow cations to move through with transport number, $\overline{t_{+}}=\hat{t_{+}}=1$. The bulk transport number reflects the mobility of $\mathrm{Cu}^{2+}$ compared to $\mathrm{SO}_{4}^{2-}, t_{+}=D_{+} /\left(D_{+}+D_{-}\right)=0.4$ [34]. This is the part of the current that is carried by cations in the bulk. Due to the slab symmetry the concentration only changes perpendicular to the membrane, where $x=0$ at the membrane and $x=L$ at the anode.

The steady-state condition of the model is a constant gradient throughout the whole cell. The gradient is linear proportional to the applied current. The limiting current arises if $c_{\text {mem }}=0$. The value of the limiting current density can also be predicted with Peers' equation [34],

$$
i_{\lim }=\frac{z F D}{\left(\overline{t_{+}}-t_{+}\right)} \frac{c_{\delta}}{\delta}
$$

where $\delta$ represents the thickness of the stagnant diffusion layer and $c_{\delta}$ the concentration at the end of this layer. For $\delta=2 \mathrm{~mm}$ and $c_{\delta}=2 c_{0}=20 \mathrm{mM}$ and ideal boundaries a limiting current density of $2.7 \mathrm{~A} / \mathrm{m}^{2}$ is obtained.

\section{RESULTS AND DISCUSSION}

The results and discussion of this work are separated into three parts. First, the differences in steady-state dynamics are discussed. Only in the co-orientation RB occurs. It reduces the ion concentration polarization (ICP) and avoids or diminishes electroconvection that occurs via the electrokinetic instability (EKI), thereby decreasing the electrolyte resistance compared to that seen with co-orientation. Second, the transition time of both types of convection is determined and shows the agreement between experiment (electrical and flow) and the theory that predicts when EKI can occur. Third, concentration measurements verify the theory further and a numerical 1D framework is constructed that predicts the onset of RB. Different current regimes are identified in which the interplay between the two types of convection is fundamentally different. 

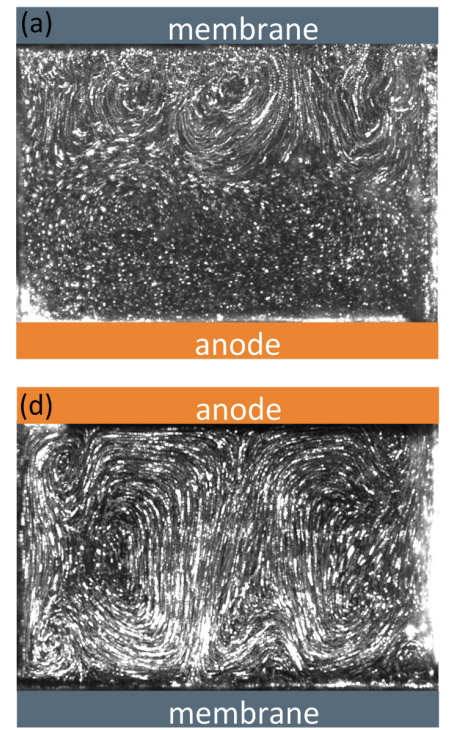
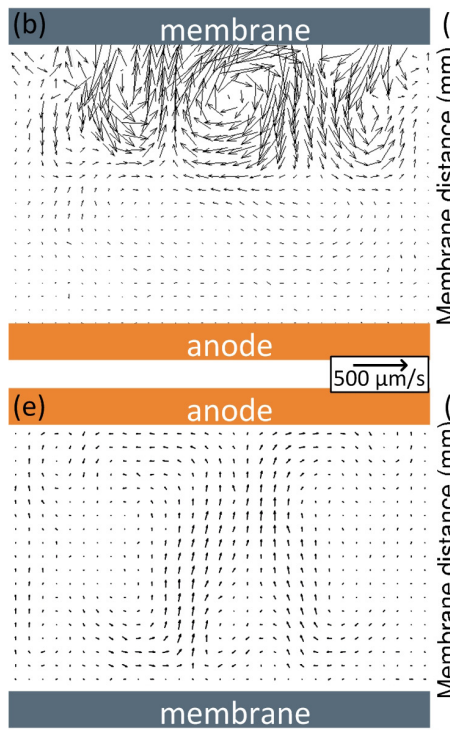
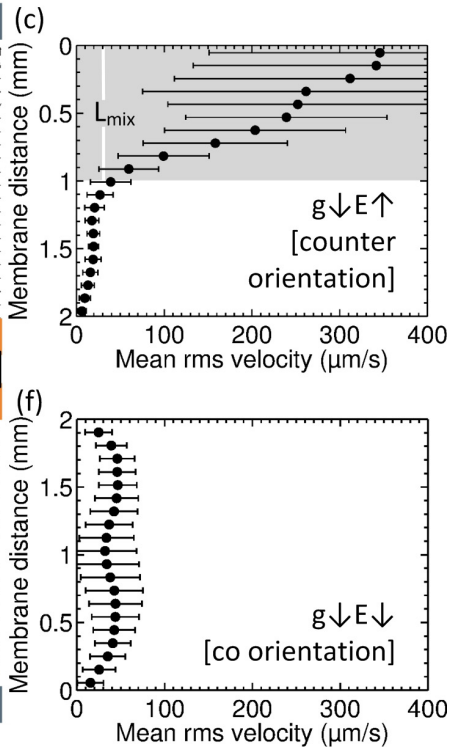

FIG. 3. (a) Particle path lines over 0.7 s. (d) Particle path lines over 5 s. (b),(e) Average velocity field over 0.1 s. (c),(f) Distribution of the mean r.m.s. velocity of a row of 31 vectors with standard deviation as a function of the height in the reservoir for different orientations. Data from $29.6 \mathrm{~A} / \mathrm{m}^{2} \approx 11 i_{\lim }, 10 \mathrm{mM} \mathrm{CuSO}_{4}, t=300$ $s$ in counterorientation (a)-(c) and co-orientation (d)-(f).

\section{A. Steady state}

In counterorientation, currents above $i_{\text {lim }}$ result in EKI and no RB convection was observed, as expected, as the ion-depleted fluid lies above the ion-enriched fluid [19,35]. In Fig. 3(a) we show the typical flow field next to the membrane after applying $29.6 \mathrm{~A} / \mathrm{m}^{2} \approx 11 i_{\text {lim }}$ when the mixing layer is fully developed $(t=300 \mathrm{~s})$. The top half of the cell shows electroconvective mixing, while the bottom half is relatively stagnant, although it clearly shows secondary vortex motion [36]. The boundary of the mixing layer is taken as the distance from the membranes where the mean r.m.s. velocity is $10 \%$ of the maximum [see Fig. 3(c)]. In Fig. 4 the mean r.m.s. velocity within the mixing layer and the applied voltage difference are shown with respect to time, which reflect the strong chaotic fluctuations in the velocity and applied voltage. The fluctuations are not a result of measurement error, since the temporal resolution of both electrical and flow measurements was sufficient. Careful analysis did not reveal a correlation between the signals. It has to be noted that the voltage is applied over the full volume, while the velocity field is extracted from a cross section. Combining the average additional voltage, $\Delta V_{\text {olc }}=3.4 \mathrm{~V}$, with the average mixing size, $L_{\text {mix }} \approx 1 \mathrm{~mm}$, estimates the conductivity of the mixing layer, $\sigma=L_{\text {mix }} i / \Delta V_{\text {olc }} \approx 9 \times 10^{1} \mu \mathrm{S} / \mathrm{cm}$. The conductivity of the mixing layer has a value similar to that found for smaller, lower intensity vortices observed in our previous work [3].

When the system is rotated $180^{\circ}$ (to co-orientation, i.e., buoyancy unstable) substantially different behavior is observed. Flow occurs in the whole cell [see Fig. 3(c)]. Fluid motion mixes the high concentration at the anode with the low concentration at the membrane. ICP diminishes and the resistance decreases towards the initial ohmic resistance, as shown in Fig. 4(a). Gravitational or Rayleigh-Bénard convection occurs if the destabilizing buoyancy forces overcome stabilizing viscous forces $[21,37,38]$. This ratio is expressed by the nondimensional Rayleigh number,

$$
\mathrm{Ra}=\frac{M \Delta c g L^{3}}{\mu D},
$$



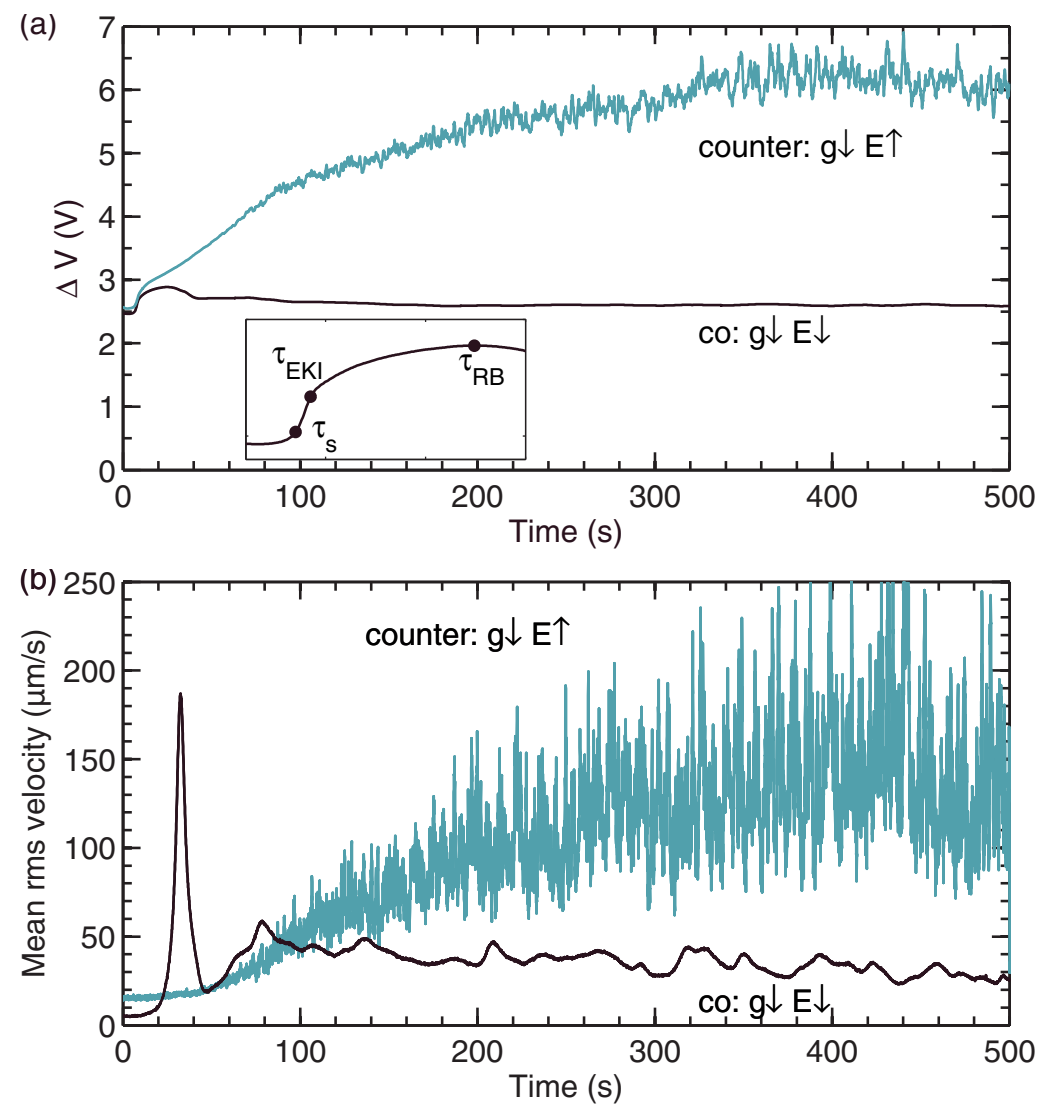

FIG. 4. The voltage drop, $\Delta V$, (a) and mean r.m.s. velocity of the PIV vectors in the full cell (b) versus time for $29.6 \mathrm{~A} / \mathrm{m}^{2}$ in $10 \mathrm{mM} \mathrm{CuSO}_{4}$ in co- and counterorientation. The inset in (a) shows the electrical transition times in the co-orientation.

where for our system, $M=0.1567 \mathrm{~kg} / \mathrm{mol}$ is the molar mass of the $\mathrm{CuSO}_{4}, g=9.81 \mathrm{~m} / \mathrm{s}^{2}$ is the gravitational constant, $\mu=1.002 \times 10^{-9} \mathrm{~kg} / \mathrm{m} / \mathrm{s}$ is the dynamic viscosity of water, and $D=$ $D_{\text {CuSO4 }}=8.55 \times 10^{-10} \mathrm{~m}^{2} / \mathrm{s}$ is the diffusion coefficient of copper sulfate.

The $\mathrm{RB}$ instability is found to occur if $\mathrm{Ra}>\mathrm{Ra}_{\mathrm{c}}$. The critical Rayleigh number depends on the system parameters but is on the order of $\mathrm{Ra}_{\mathrm{c}}=1000$, as in Ref. [19], which concerned a similar electrolyte system with flat sheet copper electrodes in $\mathrm{CuSO}_{4}$ solution. This value is also in agreement to other numerical work on the effect of nonlinear gradients between two rigid boundaries (no slip) with constant flux and constant concentration boundary values [39]. For the limiting current condition $(L=2 \mathrm{~mm}, \Delta c=20 \mathrm{mM})$ the Rayleigh number is $\mathrm{Ra} \simeq 2.9 \times 10^{5}$. The experimental observation of RB convection is therefore expected above the limiting current and agrees with other work [26,38]. Rayleigh-Bénard convection is also observed at underlimiting currents. RB flow brings denser (ion-rich) fluid down to the membrane, while less dense (ion depleted) fluid moves away. This balances the anion migration away from the membrane and diminishes the degree of ion depletion.

At $i=8 i_{\mathrm{lim}}$ and higher current densities, a small layer with EKI is present at the membrane while $\mathrm{RB}$ convection dominates the rest of the cell. The EKI layer size $O(100 \mu \mathrm{m})$ is larger at higher currents. The enhanced-ion transport caused by RB is not sufficient to sustain the high current demand and avoid depletion of the interface. Movies showing the development of vortices in the system, along with the electrical response, for different configurations as well as of concentration profile 

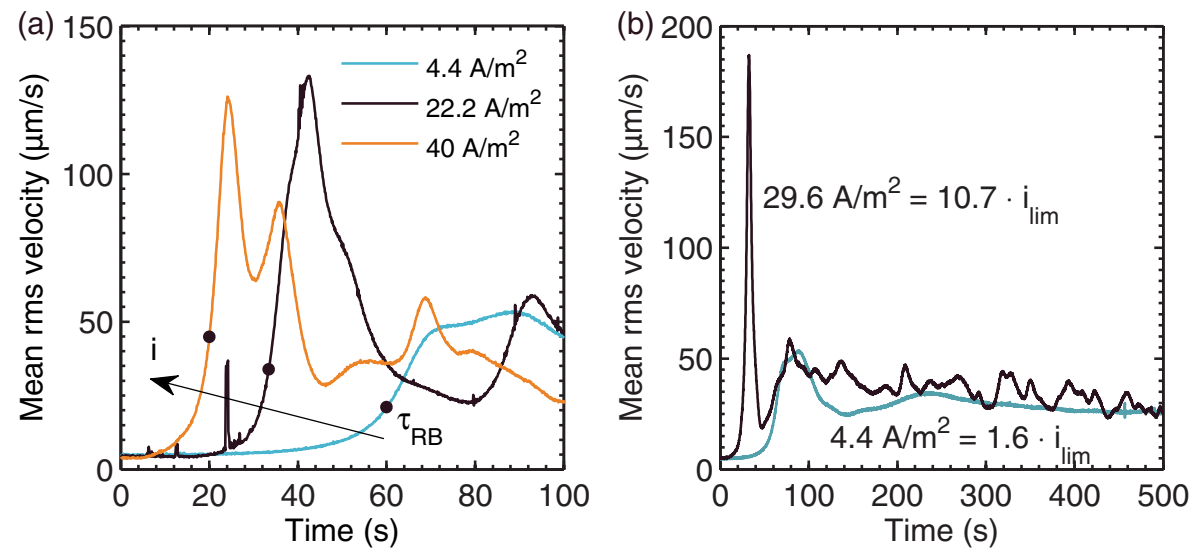

FIG. 5. (a) The growth of the mean r.m.s. velocity occurs earlier $\left(\tau_{\mathrm{RB}}\right)$ and faster at higher currents. (b) The peak velocity increases with increased current, but final average velocity seems independent of current. At higher currents there is larger fluctuation.

(fluorescence lifetime) vs time are provided in the Supplemental Material [40]. These observations confirm the numerical predictions of Karatay et al. [28]. It could not be determined in our case if the EKI vortices stayed counterrotating [9] or the RB flow caused corotating [41] or even helical [15] vortices.

\section{B. Transition times}

The onset of the instabilities provides an understanding in the coupling of both types of convection. RB mixing starts when the concentration gradient becomes buoyancy unstable and mixes the whole volume [20,21]. EKI mixing starts at the membrane and occurs after the membrane surface becomes depleted and an overvoltage is applied [6,7,11]. The time for $c_{\mathrm{mem}}=0$ can be determined from the diffusion-migration model. For an infinitely large system, $L \rightarrow \infty$, an analytical solution can be derived known as Sand's transition time [42]:

$$
\tau_{s}=\frac{\pi D}{4}\left(\frac{c_{0} z F}{\overline{t_{+}}-t_{+}}\right)^{2} \frac{1}{i^{2}} .
$$

For $c(L=1 \mathrm{~mm})=c_{0}$ this agrees with Sand's equation when the diffusion layer is smaller than $L$. It was verified that at the lowest current $\left(4.4 \mathrm{~A} / \mathrm{m}^{2}=1.6 i_{\mathrm{lim}}\right)$ the numerical depletion time is similar to $\tau_{\mathrm{s}}$. An analytical function for the transition time that is also valid at even lower currents can be found in Ref. [43].

From the chronopotentiometric measurements response, the time for ion depletion at the membrane interface is extracted, since it causes a jump in resistance [3,44]. The inset in Fig. 4(a) shows the electrical signature of the transitions. The depletion time is usually taken as the base of voltage jump [44], or the point with maximal gradient [45]. To extract the value of the voltage jump in a consistent way, the depletion time, $\tau_{\mathrm{s}}$, is taken as the first point where the rate of change of the gradient is maximum $\left[\partial_{t}^{3} V(t)=0\right]$. The end of the voltage jump and start of EKI, $\tau_{\mathrm{EKI}}$, is taken as the second point where $\partial_{t}^{3} V(t)=0$. The electrical RB transition time, $\tau_{\mathrm{RB}}$, is taken as the moment the RB mixing decreases ICP and overall resistance $\left[\partial_{t} V(t)=0\right]$.

From the simultaneous flow measurements, the start of EKI $\tau_{\mathrm{EKI}}$ is taken when the first particle movement next to the membrane appears. The flow signature of RB convection, $\tau_{\mathrm{RB}}$, is taken as the instant the full cell velocity suddenly jumps up. Figure 5(a) shows the root mean square (r.m.s.) velocity of the vector field of the whole membrane compartment as measured with PIV. After the concentration gradient becomes unstable RB convection starts and the r.m.s. velocity increases 

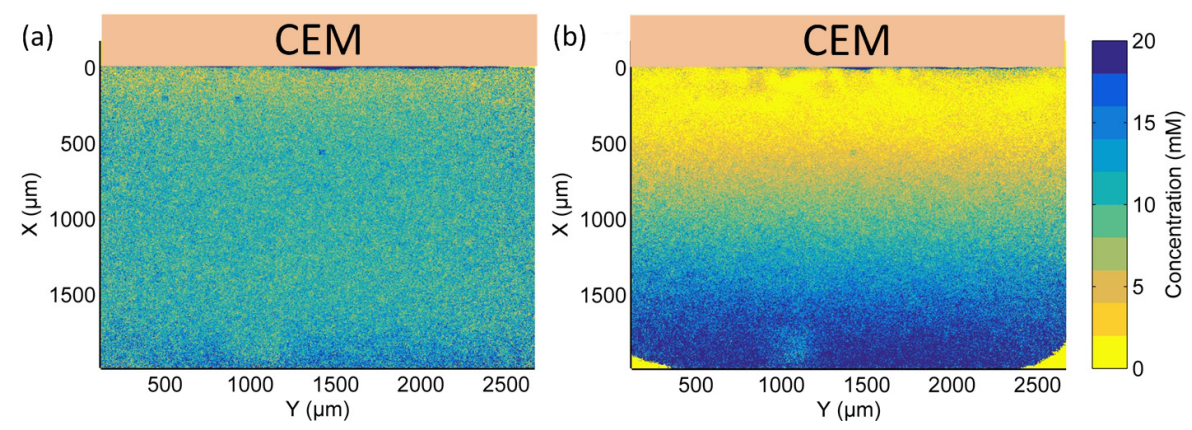

FIG. 6. Images of the FLIM lifetime of each pixel color coded from blue $2.5 \mathrm{~ns}$ to yellow 4.1 ns. The cation-exchange membrane is at the top and $i=3.3 i_{\mathrm{lim}}=8.9 \mathrm{~A} / \mathrm{m}^{2}$. (a) $t=45-60 \mathrm{~s}$; (b) $t=825-840 \mathrm{~s}$.

steeply. The base of the velocity peak is taken as the moment the increase in speed is highest $\left[\partial_{t}^{3} v_{\mathrm{rms}}(t)=0\right]$. This is the inflection point of the first derivative and local maximum of the second derivative.

The velocity fluctuation at the beginning at higher currents, $i>6.4 i_{\lim }=18 \mathrm{~A} / \mathrm{m}^{2}$, is caused by mixing via EKI at the membrane. Although the velocity within the EKI mixing layer is high, the full cell r.m.s. velocity is low, due to the fact the layer is initially small. A higher current shows a higher peak velocity and an earlier onset time. Gradients build up faster, and the concentration difference between the membrane and the anode becomes higher. After mixing starts, gradients are reduced and the velocity drops. Even after $500 \mathrm{~s}$ [Fig. 5(b)], the velocity still decreases.

\section{Concentration profile development}

In counterorientation, the concentration profile development was determined using FLIM. In Fig. 6 two images of the concentration distribution in the anode compartment, as determined from FLIM measurements, are displayed. They show the degree of concentration polarization at $t=45-60 \mathrm{~s}$ and $t=825-840 \mathrm{~s}$ for applying $i=3.3 i_{\mathrm{lim}}=8.9 \mathrm{~A} / \mathrm{m}^{2}$ inside a $10 \mathrm{mM} \mathrm{CuSO}_{4}$ solution with $2.5 \mu \mathrm{M}$ Alexa dye. From these images, a representative 1D concentration profile is calculated, as shown in Fig. 7(a). Each data point is obtained from averaging the lifetime over 100 pixels (along membrane in the center) by 20 pixels (normal to membrane).

Using the numerical model previously described, the concentration profile between the membrane and the anode is predicted, as shown in Fig. 7(a). The experimental system displays a diffusion layer and a mixing layer with a concentration, $c_{\text {mix }}=0.1 c_{0}=1 \pm 0.2 \mathrm{mM}$. This is in line with the previous postulate that the overlimiting resistance, $R_{\text {olc }}=\Delta V_{\text {olc }} /\left(i A_{\text {mem }}\right)$, is primarily characterized by a low-concentration (high-resistance) layer [3].

In co-orientation, the model can be used to predict when the boundary layer becomes unstable. The flow observations show the start of half-cell RB vortices, most pronounced at the anode, with much faster full cell motion starting quickly after. To estimate when the half cell becomes unstable, $\mathrm{Ra}>\mathrm{Ra}_{\mathrm{c}}$, a characteristic length is chosen from the nonlinear concentration gradient. For this, we take the distance between the membrane or anode and the point where the concentration gradient is $10 \%$ of the maximum value. In Fig. 7(b) this characteristic length, $L$, together with the concentration difference, $\Delta c$, is shown. With these values the Rayleigh number of the boundary layer is calculated, which is plotted against time in Fig. 7(c). From this line the time when $\mathrm{Ra}>1000$ is determined, which is taken as the numerical RB transition time, $\tau_{\mathrm{RB}}$.

An overview of all co-orientation transition times is shown in Fig. 7(d). The electrical and flow EKI transition times, $\tau_{\mathrm{EKI}}$, are similar and match well with the theoretical depletion time. The EKI onset thus follows a -2 power law with the current as seen in the Sand's equation and found in many other experiments $[3,44]$. The transition time for the RB convection decays slower with increased 

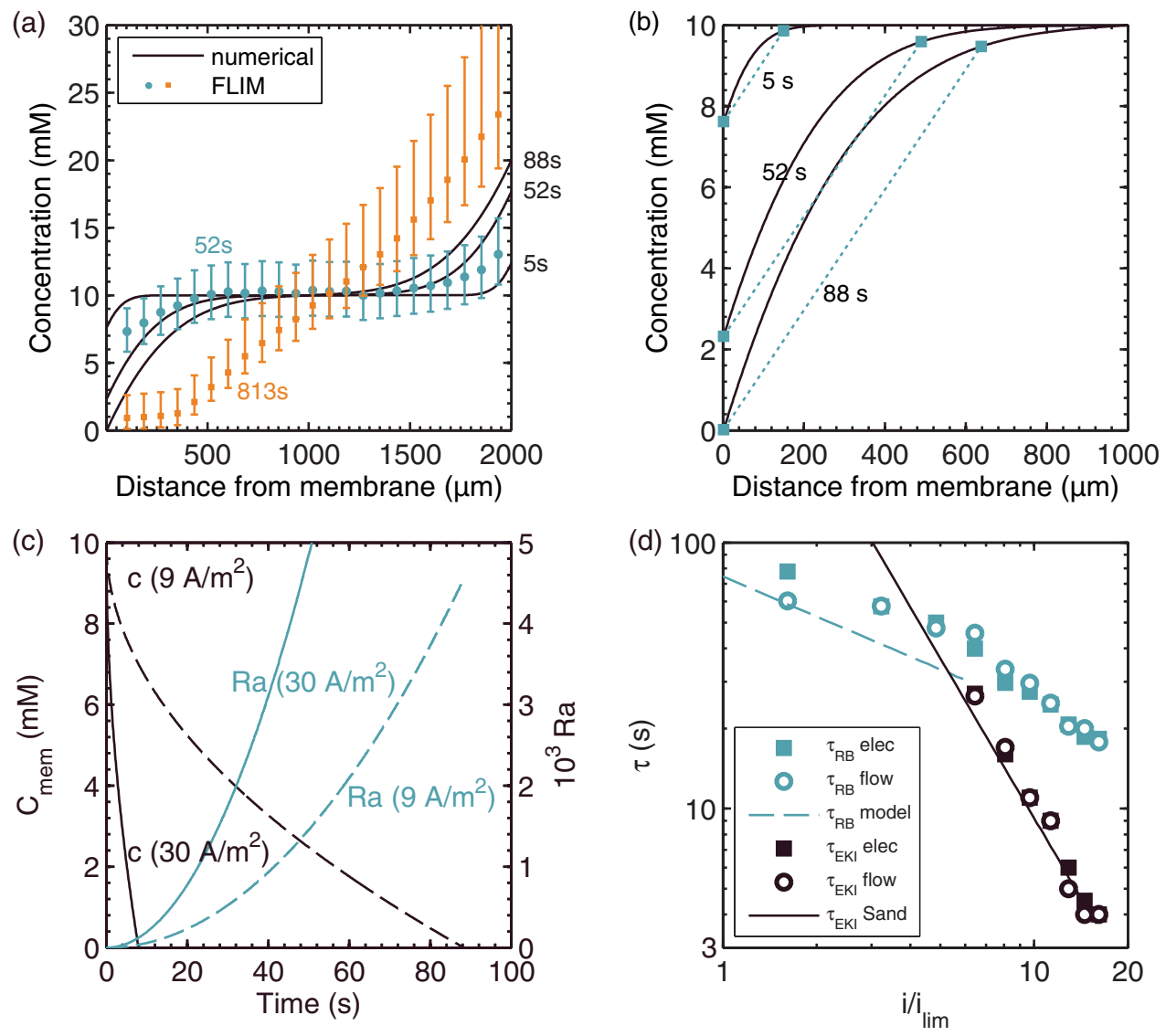

FIG. 7. (a) The numerical (black line) and experimental concentration profile (FLIM). At overlimiting current (812 s) the mixing layer has a low concentration. (b) The numerically estimated concentration profile (black solid line) for $i=8.9 \mathrm{~A} / \mathrm{m}^{2}=3.3 i_{\mathrm{lim}}$ at different times. The green dotted line shows the characteristic length and concentration difference that are used to determine Ra. $t=5 \mathrm{~s}$ gives $\mathrm{Ra}=14 . t=52 \mathrm{~s}$ gives $\mathrm{Ra}=1.5 \times 10^{3} . t=5 \mathrm{~s}$ gives $\mathrm{Ra}=4.5 \times 10^{3}$. (c) The numerical prediction of the membrane concentration and the Rayleigh number of the boundary layer in time. (d) The experimental transition times (points) vs the applied current scaled to the limiting current in log-log scale. The lines show the numerical prediction of onset of RB convection and EKI mixing.

current. This leads to two regimes, one where RB convection occurs before EKI and one where EKI starts first. The presented numerical model predicts a decay of power $\sim-0.5$, which comes close to the actual data. If EKI starts, this hastens the onset of RB convection and the data deviate from this -0.5 power prediction.

\section{CONCLUSION}

In summary, we have experimentally characterized the influence and coupling of buoyancy effects (RB, Rayleigh-Bénard) and the EKI in overlimiting current conditions. In counterorientation, which is buoyancy stable, we identify an EKI mixing layer and an almost stagnant diffusion layer. Our concentration and velocity measurements show the high OLC resistance is a result of the low concentration in the mixing layer. In co-orientation, which is buoyancy unstable, RB convection eventually dominates over EKI mixing. Our model and experimental data on the onset of both types 


\section{INFLUENCE OF RAYLEIGH-BÉNARD CONVECTION ON ...}

of convection are in agreement. For $i<5 i_{\text {lim }}$ the RB onset time is shorter compared to the Sand transition time, and RB convection prevents ion depletion at the membrane. We find that EKI starts before RB if $i>5 i_{\text {lim }}$ and that this accelerates the onset of RB due to the disturbance of the depletion layer by EKI. At currents above $i>7 i_{\text {lim }}$ EKI stays present at the membrane interface, indicating that the RB convection cannot avoid depletion. The mixing of the full cell decreases the resistance to near initial values. This mixing causes higher ion transport than diffusion allows for at limiting current conditions, which demonstrates the potentially large impact RB instabilities could have on the performance of electrodialysis systems.

\section{ACKNOWLEDGMENTS}

We thank E. Karatay, A. Mani, and M. Wessling for fruitful discussions. This work was performed in the cooperation framework of Wetsus, European Centre of Excellence for Sustainable Water Technology (www.wetsus.eu). Wetsus is co-funded by the Dutch Ministry of Economic Affairs and Ministry of Infrastructure and Environment, the Province of Fryslân, the Northern Netherlands Provinces, and University Campus Fryslân. The authors would like to thank the participants of the research theme Biomimetic membranes for the discussions and financial support. This research was also financially supported by the European Research Council, under ERC Starting Grant No. 307342-TRAM.

[1] V. V. Nikonenko, A. V. Kovalenko, M. K. Urtenov, N. D. Pismenskaya, J. Han, P. Sistat, and G. Pourcelly, Desalination at overlimiting currents: State-of-the-art and perspectives, Desalination 342, 85 (2014).

[2] H. C. Chang, E. A. Demekhin, and V. S. Shelistov, Competition between Dukhin's and Rubinstein's electrokinetic modes, Phys. Rev. E 86, 046319 (2012).

[3] J. C. de Valença, R. M. Wagterveld, R. G. H. Lammertink, and P. A. Tsai, Dynamics of microvortices induced by ion concentration polarization, Phys. Rev. E 92, 031003 (2015).

[4] T. A. Zangle, A. Mani, and J. G. Santiago, On the propagation of concentration polarization from microchannel- nanochannel interfaces. Part II: Numerical and experimental study, Langmuir 25, 3909 (2009).

[5] J. J. Krol, M. Wessling, and H. Strathmann, Concentration polarization with monopolar ion exchange membranes: currentvoltage curves and water dissociation, J. Membr. Sci. 162, 145 (1999).

[6] S. S. Dukhin, Electrokinetic phenomena of the second kind and their applications, Adv. Colloid Interface Sci. 35, 173 (1991).

[7] I. Rubinstein and B. Zaltzman, Electro-osmotically induced convection at a permselective membrane, Phys. Rev. E 62, 2238 (2000).

[8] S. Kim, Y.-C. Wang, J. Lee, H. Jang, and J. Han, Concentration Polarization and Nonlinear Electrokinetic Flow near a Nanofluidic Channel, Phys. Rev. Lett. 99, 044501 (2007).

[9] S. Rubinstein, G. Manukyan, A. Staicu, I. Rubinstein, B. Zaltzman, R. Lammertink, F. Mugele, and M. Wessling, Direct Observation of a Nonequilibrium Electro-Osmotic Instability, Phys. Rev. Lett. 101, 236101 (2008).

[10] G. Yossifon and H.-C. Chang, Selection of Nonequilibrium Overlimiting Currents: Universal Depletion Layer Formation Dynamics and Vortex Instability, Phys. Rev. Lett. 101, 254501 (2008).

[11] C. L. Druzgalski, M. B. Andersen, and A. Mani, Direct numerical simulation of electroconvective instability and hydrodynamic chaos near an ion-selective surface, Phys. Fluids 25, 110804 (2013).

[12] E. A. Demekhin, N. V. Nikitin, and V. S. Shelistov, Direct numerical simulation of electrokinetic instability and transition to chaotic motion, Phys. Fluids 25, 122001 (2013).

[13] V. S. Pham, Z. Li, K. M. Lim, J. K. White, and J. Han, Direct numerical simulation of electroconvective instability and hysteretic current-voltage response of a permselective membrane, Phys. Rev. E 86, 046310 (2012). 
[14] E. A. Demekhin, N. V. Nikitin, and V. S. Shelistov, Three-dimensional coherent structures of electrokinetic instability, Phys. Rev. E 90, 013031 (2014).

[15] S. V. Pham, H. Kwon, B. Kim, J. K. White, G. Lim, and J. Han, Helical vortex formation in threedimensional electrochemical systems with ion-selective membranes, Phys. Rev. E 93, 033114 (2016).

[16] R. Kwak, V. S. Pham, K. M. Lim, and J. Han, Shear Flow of an Electrically Charged Fluid by Ion Concentration Polarization: Scaling Laws for Electroconvective Vortices, Phys. Rev. Lett. 110, 114501 (2013).

[17] J. Balster, M. H. Yildirim, D. F. Stamatialis, R. Ibanez, R. G. H. Lammertink, V. Jordan, and M. Wessling, Morphology and microtopology of cation-exchange polymers and the origin of the overlimiting current, J. Phys. Chem. B 111, 2152 (2007).

[18] S. M. Davidson, M. Wessling, and A. Mani, On the dynamical regimes of pattern-accelerated electroconvection, Sci. Rep. 6, 1 (2016).

[19] B. Baranowski and A.L. Kawczyński, Experimental determination of the critical rayleigh number in electrolyte solutions with concentration polarization, Electrochim. Acta 17, 695 (1972).

[20] W. J. Ward and O. H. Le Blanc, Rayleigh-Benard Convection in an Electrochemical Redox Cell, Science (Washington, DC, U. S.). 225, 1471 (1984).

[21] V. M. Volgin and A. D. Davydov, Natural-convective instability of electrochemical systems: A review, Russ. J. Electrochem. 42, 567 (2006).

[22] V Fleury, J.-N. Chazalviel, and M Rosso, Theory and Experimental Evidence of Electroconvection Around Electrochemical Deposits, Phys. Rev. Lett. 68, 2492 (1992).

[23] M. Rosso, J.-N. Chazalviel, V. Fleury, and E. Chassaing, Experimental evidence for gravity induced motion in the vicinity of ramified electrodeposits, Electrochim. Acta 39, 507 (1994).

[24] G. Marshall, E. Mocskos, F. V. Molina, and S. Dengra, Three-dimensional nature of ion transport in thin-layer electrodeposition, Phys. Rev. E 68, 021607 (2003).

[25] C. W. Tobias, M. Eisenberg, and C. R. Wilke, Diffusion and convection in electrodialysis-A theoretical review, J. Electrochem. Soc. 99, 359 (1952).

[26] H. W. Rösler, F. Maletzki, and E. Staude, Ion transfer across electrodialysis membranes in the overlimiting current range: Chronopotentiometric studies, J. Membr. Sci. 72, 171 (1992).

[27] V. I. Zabolotsky, V. V. Nikonenko, and N. D. Pismenskaya, On the role of gravitational convection in the transfer enhancement of salt ions in the course of dilute solution electrodialysis, J. Membr. Sci. 119, 171 (1996).

[28] E. Karatay, M. B. Andersen, M. Wessling, and A. Mani, Coupling between Buoyancy Forces and Electroconvective Instability near Ion-Selective Surfaces, Phys. Rev. Lett. 116, 194501 (2016).

[29] J. Hohlbein, M. Steinhart, C. Schiene-Fischer, A. Benda, M. Hof, and C. G. Hübner, Confined diffusion in ordered nanoporous alumina membranes, Small 3, 380 (2007).

[30] G. Scalia and F. Scheffold, Lifetime of fluorescent dye molecules in dense aqueous suspensions of polystyrene nanoparticles, Opt. Express 23, 29342 (2015).

[31] J. R. Lakowicz, Principles of Fluorescence Spectroscopy, 3rd ed. (Springer, New York, 2013).

[32] P. Sistat and G. Pourcelly, Chronopotentiometric response of an ion-exchange membrane in the underlimiting current-range. Transport phenomena within the diffusion layers, J. Membr. Sci. 123, 121 (1997).

[33] P. Vanýsek, in CRC Handbook of Chemistry and Physics, 97th ed., edited by W. M. Haynes (CRC Press, Boca Raton, FL, 2016).

[34] V. V. Nikonenko, N. D. Pismenskaya, E. I. Belova, P. Sistat, P. Huguet, G. Pourcelly, and C. Larchet, Intensive current transfer in membrane systems: Modelling, mechanisms and application in electrodialysis, Adv. Colloid Interface Sci. 160, 101 (2010).

[35] F. Maletzkl, H. Rdsler, E. Staude, F. Maletzki, H.-W. Rösler, and E. Staude, Ion transfer across electrodialysis membranes in the overlimiting current range: Stationary voltage current characteristics and current noise power spectra under different conditions of free convection, J. Membr. Sci. 71, 105 (1992).

[36] S. Hanasoge and F. J. Diez, Vortex Chain Formation in Regions of Ion Concentration Polarization, Lab-on-a-Chip 15, 3549 (2015). 


\section{INFLUENCE OF RAYLEIGH-BÉNARD CONVECTION ON ...}

[37] M. Rosso, E. Chassaing, and J.-N. Chazalviel, Role of buoyancy in the onset of dendritic growth in thin layer electrodeposition, Phys. Rev. E 59, 3135 (1999).

[38] N. D. Pismenskaya, V. V. Nikonenko, E. I. Belova, G. Yu. Lopatkova, Ph. Sistat, G. Pourcelly, and K. Larshe, Coupled convection of solution near the surface of ion-exchange membranes in intensive current regimes, Russ. J. Electrochem. 43, 307 (2007).

[39] E. M. Sparrow, R. J. Goldstein, and V. K. Jonsson, Thermal instability in a horizontal fluid layer: Effect of boundary conditions and non-linear temperature profile, J. Fluid Mech. 18, 513 (1964).

[40] See Supplemental Material at http://link.aps.org/supplemental/10.1103/PhysRevFluids.2.033701 for experimental movies.

[41] R. Kwak, G. Guan, W K. Peng, and J. Han, Microscale electrodialysis: Concentration profiling and vortex visualization, Desalination 308, 138 (2013).

[42] A. J. Bard and L. R. Faulkner, Electrochemical Methods: Fundamentals and Applications, 2nd ed. (Wiley, New York, 2001).

[43] M. Van Soestbergen, P. M. Biesheuvel, and M. Z. Bazant, Diffuse-charge effects on the transient response of electrochemical cells, Phys. Rev. E 81, 021503 (2010).

[44] J. J. Krol, M. Wessling, and H. Strathmann, Chronopotentiometry and overlimiting ion transport through monopolar ion exchange membranes, J. Membr. Sci. 162, 155 (1999).

[45] S. A. Mareev, D. Yu Butylskii, N. D. Pismenskaya, and V. V. Nikonenko, Chronopotentiometry of ion-exchange membranes in the overlimiting current range. Transition time for a finite-length diffusion layer: Modeling and experiment, J. Membr. Sci. 500, 171 (2016). 